\title{
Circulação de ideias em eventos da História da educação matemática
}

\author{
Circulation of ideas on History of mathematics \\ education events
}

Circulación de ideas en eventos de la Historia de la educación matemática

YOHANA TAISE HOFFMANN

DAVID ANTONIO DA COSTA

\section{Resumo}

Consideramos a História da educação matemática (Hem) como um campo científico que possui como elementos constitutivos os grupos de pesquisas, as produções científicas, como teses e dissertações, as disciplinas que contribuem para a autonomia e estabilidade do próprio campo e as comunicações científicas, como os eventos e as revistas. Mobilizamos a sociologia da educação de Pierre Bourdieu como referencial teórico, principalmente na definição do conceito de campo. Dessa forma, o presente artigo tem por objetivo apresentar sócio historicamente a circulação de ideias a partir dos eventos e as revistas científicas do campo da Hem. Apresentamos o International Congress on Mathematical Education (ICME), em seguida a revista International Journal on the History of Mathematics Education (IJHME), que circulou entre os anos de 2006 e 2016. A partir da mobilização da comunidade internacional de pesquisadores que investigam a Hem, foi criado o International Conference on the History of Mathematics Education (ICHME), logo em seguida o Congresso Iberoamericano de História da Educação Matemática (CIHEM) e, no Brasil, o Encontro Nacional de Pesquisa em História da Educação Matemática (ENAPHEM). Entre todas

\footnotetext{
a Universidade Federal de Santa Catarina (UFSC), Florianópolis, SC, Brasil. Mestre em Educação Científica e Tecnológica, e-mail: yohana.thc@gmail.com

b Universidade Federal de Santa Catarina (UFSC), Florianópolis, SC, Brasil. Doutor em Educação Matemática, e-mail: david.costa@ufsc.br
} 
as revistas atualmente que contribuem para a circulação de ideias elencamos a Revista UNIÓN, intitulada Historia Social de la Educación Matemática en Iberoamérica e a Revista HISTEMAT, intitulada Revista de História da Educação Matemática. Os espaços que a Hem vem ocupando contribuem para o processo de reconhecimento, legitimação, socialização e circulação de ideias do próprio campo.

Palavras-chave: Campo científico. Comunicação científica. Eventos e Revistas científicas. Pierre Bourdieu. Sociologia da educação.

\begin{abstract}
We consider the History of mathematics education (Hme) as a scientific field which has as constitutive elements, research groups, scientific productions (e.g. thesis and dissertations), school and university subjects that contribute to the autonomy and stability of the field itself and scientific communications, such as specialised events and journals. Pierre Bourdieu's sociology of education is mobilised as the theoretical framework, mainly on his definition of field concept. In this sense, this paper aims to present the circulation of ideas on a sociohistorical point of view through scientific events and journals on Hme field. We present the International Congress on Mathematical Education (ICME) and then the International Journal on the History of Mathematics Education (IJHME), that circulated between years 2006 and 2016. Based on the mobilisation of the international community of researchers investigating Hme, the International Conference on the History of Mathematics Education (ICHME) was launched, shortly thereafter the Congresso IberoAmericano de História da Educação Matemática (CIHEM) and, in Brazil, the Encontro Nacional de Pesquisa em História da Educação Matemática (ENAPHEM). Among all journals that currently contribute to the circulation of ideas, we list Revista UNIÓN, entitled Historia Social de la Educación Matemática en Iberoamérica and the Revista HISTEMAT, entitled Revista de História da Educação Matemática. The spaces that Hme has been occupying contribute to the process of recognition, legitimation, socialisation and circulation of ideas from the field itself.
\end{abstract}

Keywords: Pierre Bourdieu. Scientific communication. Scientific events and journals. Scientific field. Sociology of education.

\title{
Resumen
}

Consideramos la Historia de la educación matemática (Hem) como un campo científico que tiene como elementos constitutivos grupos de investigación, producciones científicas como tesis de doctorado y de maestría, las disciplinas que contribuyen a la autonomía y estabilidad del campo en sí y las comunicaciones científicas, como eventos y revistas. Movilizamos la sociología de la educación de Pierre Bourdieu como marco teórico, principalmente en la definición del concepto de campo. Por lo tanto, este artículo tiene 
como objetivo presentar históricamente la circulación de ideas de eventos y revistas científicas en el campo de Hem. Presentamos el the International Congress on Mathematical Education (ICME), seguido de la Revista the International Journal on the History of Mathematics Education (IJHME), que circuló entre 2006 y 2016. Desde la movilización de la comunidad internacional de investigadores que investigan Hem, se creó la International Conference on the History of Mathematics Education (ICHME), seguida del Congreso Iberoamericano de Historia de la Educación Matemática (CIHEM) y en Brasil lo Encontro Nacional de Pesquisa em História da Educação Matemática (ENAPHEM). Entre todas las revistas que actualmente contribuyen a la circulación de ideas, enumeramos Revista UNIÓN, titulada Historia Social de la Educación Matemática en Iberoamérica y Revista HISTEMAT, titulada Revista de História da Educação Matemática. Los espacios que Hem ha estado ocupando contribuyen al proceso de reconocimiento, legitimación, socialización y circulación de ideas desde el campo mismo.

Palabras clave: Campo científico. Comunicación científica. Eventos y revistas científicas. Pierre Bourdieu. Sociología de la educación.

\title{
Introdução
}

\begin{abstract}
De qualquer maneira, apesar da relevância das novas formas de comunicação, o meio mais eficaz de disseminação continua sendo o antigo, ou seja, o encontro com as pessoas (BURKE, 2016, p. 114).
\end{abstract}

Iniciamos esse artigo com o excerto de Burke (2016) a respeito da disseminação de conhecimentos, mesmo havendo cartas, periódicos, livros, e com as novas tecnologias de informação e comunicação, por meio da internet, a comunicação via e-mails e a disseminação de revistas eletrônicas, ainda o meio mais eficaz é o encontro com as pessoas.

Segundo Targino (2000, p. 10), “A comunicação científica é indispensável à atividade científica". Permite a troca contínua entre os pares e, ainda, segundo a autora, é o espaço "que favorece ao produto (produção científica) e aos produtores (pesquisadores) a necessária visibilidade e possível credibilidade no meio social em que produto e produtores se inserem" (p. 1).

Por comunicação científica compreende-se um público, um discurso e uma natureza específica. De acordo com Bueno (2010), “a comunicação científica está presente em círculos mais restritos, como eventos técnico-científicos e periódicos científicos” (p. 4). Visa, em particular, a disseminação e socialização de informações intrínsecas aos pares, "com o intuito de tornar conhecidos, na comunidade 
científica, os avanços obtidos (resultados de pesquisas, relatos de experiências, etc.) em áreas específicas ou à elaboração de novas teorias ou refinamento das existentes" (p. 5). O autor atenta para dois casos de comunicação científica, a comunicação intrapares e extrapares, que mesmo o público sendo constituído por especialistas há diferenças. Neste artigo estamos interessados na comunicação intrapares, como podemos observar no seguinte excerto:

A comunicação intrapares compreende a circulação de informações científicas, tecnológicas e de inovação entre especialistas de um campo ou de campos conexos. A comunicação extrapares diz respeito ao mesmo processo, mas tem como público-alvo especialistas que não se situam, por formação ou atuação específica, na área que é objeto da disseminação. São exemplos de comunicação intrapares a que se materializa por periódicos especializados ou por eventos científicos em áreas bem delimitadas, de tal modo que aqueles que não as freqüentam encontram dificuldades para acessá-los (BUENO, 2010, p. 9).

A partir das reflexões iniciais no XIV Congresso Nacional de Educação (EDUCERE) ${ }^{1}$, a respeito da História da educação matemática (Hem) como um campo científico, caracterizando os seus elementos constitutivos a fim de compreender a sua estrutura ${ }^{2}$, ampliamos algumas discussões ao tratar desse campo em específico. Dessa forma, temos como objetivo apresentar sócio historicamente a circulação de ideias a partir de comunicações científicas, em particular os eventos e as revistas científicas do campo da Hem.

O referencial teórico que nos embasamos é a sociologia da educação de Bourdieu (1930-2002), mobilizando principalmente a sua definição de campo. Para o autor, os diferentes campos científicos se conceituam como espaços sociais constituídos por relações de tensão e de dominação entre os agentes, considerados microcosmos "que, sob certos aspectos, constituem mundos sociais idênticos aos demais, com concentrações de poder e de capital, monopólios, relações de força, interesses egoístas, conflitos etc.” (BOURDIEU, 2001a, p. 133).

Para Bourdieu (2001b, p. 70), os campos possuem diferentes graus de autonomia, o que "significa que o sistema de forças constitutivas da estrutura do

\footnotetext{
${ }^{1}$ Realizado entre os dias 16 a 19 de setembro de 2019, na Pontifícia Universidade Católica do Paraná (PUCPR).

${ }^{2}$ Trabalho apresentado no eixo Sociologia da Educação intitulado "PROCESSO DE CONSTITUIÇÃO DE UM CAMPO CIENTÍFICO: História da educação matemática no Brasil".
} 
campo (tensão) é relativamente independente das forças que exercem sobre o campo (pressão)". Assim, ao pensar a autonomia relativa dos campos, segundo Bourdieu (2001b), é pensar que o campo “dispõe, de alguma forma, da 'liberdade' necessária para desenvolver a sua própria necessidade, a sua própria lógica, o seu próprio nomos" (p. 70, grifo do autor). Dessa forma,

A autonomia não é um dado, mas uma conquista histórica, sempre renovada. Esquecemos isto facilmente no caso das ciências da natureza, porque a autonomia está inscrita, em simultâneo, na objectividade das estruturas do campo e também nas mentes, na forma de teorias e métodos incorporados que voltaram ao estado prático (p. 70).

Ao tratar do processo de desenvolvimento de um campo científico, Bourdieu (2001b) menciona o historiador e sociólogo das ciências Yves Gingras, ao distinguir o campo em dois momentos:

[...] em primeiro lugar, a emergência de uma prática de investigação, ou seja, de agentes cuja prática assenta mais na investigação do que no ensino, e a institucionalização da investigação na universidade através da criação de condições favoráveis à produção do saber e à reprodução a longo prazo do grupo; em segundo lugar, a constituição de um grupo reconhecido como socialmente distinto e de uma identidade social, quer disciplinar, através da criação de associações científicas, quer profissional, através da criação de uma corporação: os cientistas dotam-se de representantes oficiais que lhes dão visibilidade social e que defendem os seus interesses (GINGRAS apud BOURDIEU, 2001b, p.73).

Desta maneira, elencamos como elementos que constituem um campo científico as produções científicas na área com a realização de teses e dissertações; seguido da criação de Grupos de Pesquisas (GPs); outro aspecto que contribui para a sua constituição é a divulgação e socialização das pesquisas, como os eventos científicos e as revistas temáticas; por fim, as disciplinas contribuem para a autonomia e estabilidade do próprio campo.

Apresentamos um breve panorama a respeito das produções científicas no campo da Hem no Brasil. Em um primeiro momento foi realizado uma busca, no dia 07 de julho de 2019, no Diretório dos Grupos de Pesquisa no Brasil, vinculado ao Conselho Nacional de Desenvolvimento Científico e Tecnológico (CNPq), a fim de identificar os GPs que investigam a Hem. O descritor utilizado na busca foi o 
termo exato "bistória da educaşão matemática", aplicado ao nome do grupo e linha de pesquisa, resultando em 18 GPs $^{3}$.

Em relação à produção de teses e dissertações, de acordo com Brito e Miorim (2016), no ano de 1984 tem-se a primeira dissertação na área, no Programa de Pós-Graduação em Educação da Universidade Federal do Paraná (UFPR), de Maria Antonieta Meneghini Martins, intitulada "Estudo da Evolução do Ensino secundário no Brasil e no estado do Paraná com ênfase na disciplina de Matemática". Sendo, em 1995, defendida a primeira tese de doutorado, no Programa em Educação, agora pela Universidade Estadual de Campinas (UNICAMP), de Maria Ângela Miorim, intitulado “O ensino de matemática: evolução e modernização".

As autoras supracitadas realizaram um levantamento no Brasil na área da Hem, a partir de diversas bases de dados, no período de 1984 a 2011, o qual totalizou 200 pesquisas, sendo 148 dissertações e 52 teses. A fim de verificar o crescimento desse campo científico, optamos pela realização da busca no Banco de Teses e Dissertações da Coordenação de Aperfeiçoamento de Pessoal de Nível Superior (CAPES), no dia 08 de julho de 2019, com o termo "História da educação matemática”, onde foram obtidos 232 resultados a partir do ano de 1999 ao ano de 2018, correspondendo aos níveis de Doutorado (62), Mestrado (122) e Mestrado Profissional (48). Segundo Brito e Miorim (2016), a partir do ano de 2007 é possível verificar uma tendência crescente de trabalhos defendidos. $\mathrm{Na}$ busca realizada no Banco de Teses e Dissertações da CAPES, foi possível verificar um aumento significativo a partir do ano de 2007, corroborando o afirmado pelas autoras. Outro destaque é para o ano de 2017, com 33 defesas, sendo então o ano de maior frequência.

Outro aspecto do campo da Hem, que contribui para sua constituição, são as disciplinas. Conforme Brito e Miorim (2016), a disciplina de "História da educação matemática" ofertada nos cursos de licenciatura em Matemática, foi lecionada pela primeira vez na Universidade Federal do Rio Grande do Norte (UFRN), no ano de 2001.

\footnotetext{
${ }^{3}$ Anexo o quadro com a relação dos Grupos de pesquisas. 
Segundo Hoffmann e Costa (2019), há um dossiê intitulado "História da Educação Matemática e Formação de Professores que Ensinam Matemática", realizado pela revista Cadernos de História da Educação, volume 16, n. 3, no ano de 2017, uma compilação de vários artigos que relatam experiências de implementação da Hem como disciplina nos cursos de licenciatura em Matemática. Em 2009 ocorreu a primeira oferta da disciplina em caráter obrigatório para os alunos ingressantes na Universidade Federal de Juiz de Fora (UFJF) e, posteriormente, em 2017, na Universidade Federal do Rio Grande do Sul (UFRGS). Ainda em 2017, na Universidade Federal de Santa Catarina (UFSC), foi ofertada, agora em caráter optativo, aos alunos matriculados no sétimo semestre do curso.

Dentre os demais elementos que podemos caracterizar do campo científico estão as comunicações científicas, em particular os eventos e as revistas científicas, que contribuem para a divulgação e a socialização entre os pares. Apresentamos no próximo tópico, os espaços que a Hem vem ocupando, contribuindo para o processo de reconhecimento, legitimação, socialização e circulação de ideias no campo.

\section{Comunicação científica: eventos e revistas do campo da Hem}

[...] a comunidade científica, como estrutura social que é, não pode prescindir de valores éticos e morais. Como decorrência, a comunicação científica, como parte integrante dessa estrutura, também está sujeita à interferência de prescrições que direcionam as atitudes comportamentais dos pesquisadores e portanto influenciam a produção científica (TARGINO, 2000, p. 13).

Iniciamos esse tópico com a citação de Targino (2000), a fim de desvelar e desnaturalizar os aspectos da ciência neutra. Como vimos anteriormente, o campo científico é um microcosmo, um espaço de lutas e tensões, em constantes disputas entre os pares, em conflitos internos e externos ao próprio campo. Dessa forma, os eventos e as revistas científicas são espaços de disputas entre os especialistas em um determinado campo. No entanto, segundo Bourdieu (2004), não

[...] basta reunir um grupo para produzir a reflexão científica, mas acredito que, com a condição de instaurar uma tal estrutura de troca que traga em si mesma o 
princípio de sua própria regulação, podem-se instaurar formas de reflexão que hoje não têm lugar e que podem ir além de todas as especulações de especialistas [...] e de todas as recomendações de comitês e de comissões (p. 18).

Destarte, apresentamos alguns caminhos que a Hem circulou, por meio de eventos e revista científicas. De acordo com Furinghetti e Karp (2018), em 2004 ocorreu um marco importante na Hem: o International Congress on Mathematical Education (ICME), em sua décima edição, contou com o TSG (Topic Study Group) em "history of mathematics education".

O ICME, cuja primeira edição ocorreu em 1969 na cidade Lyon, França, é realizado a cada quatro anos. O ICME 10, realizado em Copenhagen, Dinamarca, no ano de 2004, internacionalizou as discussões que investigam a Hem.

Segundo González (2018), a comunidade de pesquisadores interessados em estudar a Hem só vem aumentando e o autor elenca cinco aspectos que ilustram esse cenário. O primeiro está relacionado ao ICME 13:

1. Desenvolvimento de grupos de trabalho estáveis em eventos internacionais dedicados à história da Educação Matemática, como o TSG 24 History of the teaching and learning of mathematics del International Congress of Mathematical Education, cujos membros compartilham as descobertas e questões pendentes na história de educação matemática, além de trabalhar na busca de acordos relacionados às questões metodológicas próprias da pesquisa nesta temática (GONZÁLEZ, 2018, p. 280 , grifos do autor, tradução livre $)^{4}$.

O ICME 13, realizado no ano de 2016, em Hamburgo, Alemanha, também contou com um tópico de estudo em Hem, e os organizadores do TSG 24 foram Karp e Furinghetti (2018). A publicação dos materiais extraídos dos trabalhos do TSG 24 encontra-se no livro Researching the History of Mathematics Education, composto por 14 artigos que refletem as discussões da comunidade de pesquisadores em Hem.

O décimo quarto ICME, que seria realizado entre os dias 12 a 19 de julho de 2020, na cidade de Shanghai, China, foi adiado para o ano seguinte por conta da

\footnotetext{
4 “1. Desenvolvimiento de grupos de trabajo estables en eventos de carácter internacional dedicados a la historia de la Educación Matemática, tales como el TSG 24 History of the teaching and learning of mathematics del International Congress of Mathematical Education, cuyos miembros comparten los hallazgos y asuntos pendientes en la historia de la educación matemática así como también trabajan en la búsqueda de acuerdos relacionados con las cuestiones metodológicas propias de la investigación en esta temática" (GONZÁLEZ, 2018, p. 280, grifos do autor).
} 
Pandemia COVID-19 (COrona VIrus Disease, enquanto 19 se refere ao ano 2019, quando os primeiros casos foram divulgados). O TSG que dialoga com as pesquisas que investigam a Hem, é o TSG 55 "The history of the teaching and learning of mathematics", tendo como organizadores Wagner Rodrigues Valente e Alexander Karp. O TSG pretende reunir estudiosos interessados em pesquisas relacionadas a Hem, contribuindo para a discussão de descobertas e problemas não resolvidos, bem como de questões na metodologia de pesquisa.

Segundo Furinghetti e Karp (2018), outro acontecimento importante na socialização, divulgação e circulação de ideias no campo da Hem foi a criação da primeira revista científica, International Journal on the History of Mathematics Education, IJHME, que circulou entre os anos de 2006 e 2016. Segundo Schubring (2006), um dos principais objetivos da revista era fornecer memória ao ensino de matemática, assim como desvelar as falácias dos eventos passados, visando superar histórias nacionais, culturais e sociais desconectadas e contribuir para estabelecer temas e características comuns do desenvolvimento da instrução matemática em muitas culturas, diferenciando entre o que constitui especificidades ou particularidades nacionais e o que pode ser indicativo de tendências globais.

Esta é a primeira edição da Revista, que pretende servir como um fórum internacional para estudos acadêmicos sobre a história do ensino e educação da matemática, um campo que até então era marginalmente representado pelos periódicos existentes. Foi um grande sucesso o Topic Study Group 29, The History of Learning and Teaching Mathematics, no 10th International Congress on Mathematics Education em Copenhague, em 2004, que demonstrou a necessidade de um fórum internacional permanente e estável para tal pesquisa. Estamos, portanto, confiantes de que uma revista internacional dedicada à história da educação matemática, complementar a periódicos em educação matemática, matemática, história da matemática e história da educação, será de interesse substancial para educadores, políticos, historiadores e matemáticos (SCHUBRING, 2006, p. 1, tradução livre) $)^{5}$.

\footnotetext{
5 "This is the first issue of the Journal, which intends to serve as an international forum for scholarly studies on the history of mathematics teaching and education, a field which hitherto was marginally represented by the existing journals. It was the rousing success of the Topic Study Group 29, The History of Learning and Teaching Mathematics, at the 10th International Congress on Mathematics Education in Copenhagen in 2004, which demonstrated the need for a permanent and stable international forum for such research. We feel therefore confident that an international journal devoted to the history of mathematics education, complementary to journals in mathematics education, mathematics, the history of mathematics, and the history of education, will be of
} 
Com tópicos específicos de estudos em eventos, e principalmente após a criação da revista, os pesquisadores do campo da Hem, em nível internacional, observaram a necessidade de realizar um evento específico do campo. Assim, no ano de 2009 foi realizada a primeira International Conference on the History of Mathematics Education (ICHME), realizada de 21 a 24 de junho, na Islândia. Foi a primeira vez que uma conferência internacional foi exclusivamente dedicada a este tema. Os trabalhos abordaram questões de reflexões metodológicas, interdisciplinaridade e contextos do ensino de matemática, a internacionalização da educação, o movimento da matemática moderna e movimentos no desenvolvimento da educação matemática em diversos países (BJARNADÓT'TIR et al., 2009).

Os organizadores expressaram sua confiança no evento, dada a variedade de temas, o número de países envolvidos e a presença de acadêmicos experientes, juntamente com jovens pesquisadores, de que essa área de pesquisa continuaria a se desenvolver, incentivando a descoberta de sua própria história e de sua ampliação em diferentes contextos.

Os ICHMEs, são realizados a cada dois anos, o ICHME-2 ocorreu entre os dias 2 e 5 de outubro de 2011 em Lisboa. Mesmo com a representatividade de 35 participantes da Islândia, com 18 contribuições, neste ICHME-2 havia 40 pesquisadores, representando 18 países, com apresentação de cerca de 30 trabalhos (BJARNADÓTTIR et al., 2012).

O ICHME-3, foi realizado no Departamento de Educação da Universidade de Uppsala, Suécia, entre os dias 25 e 28 de setembro de 2013. De acordo com os organizadores, Bjarnadóttir et al. (2015), o evento contou com 35 participantes, representando 13 países, e 31 trabalhos apresentados. Destes, após as revisões pelos pares, foram publicados 26 artigos.

Para dar continuidade ao projeto dos ICHMEs, os organizadores mantêm o título original da primeira conferência, ou seja, "Dig where you stand". A frase em inglês, é o título do livro de Gräv där du står (1978) do autor sueco Sven Lindqvist. A abordagem "Diga onde você está" pode ser um lema adequado para

substantial interest to educators, policy makers, historians, and mathematicians" (SCHUBRING, 2006, p. 1). 
aqueles (historiadores, educadores e professores) que desejam entender de maneira sensível e profunda o ensino e a aprendizagem da matemática (BJARNADÓTTIR et al., 2015).

No ano de 2015, entre os dias 23 e 26 de setembro, foi realizado o ICHME4, na Academia de Ciências e na Universidade de Turim, Itália. Ao todo, o evento contou com 51 participantes de 16 países, 44 trabalhos entre relatórios de pesquisa e pôsteres (BJARNADÓTTIR et al., 2017). De acordo com Arzarello (2017), o ICHME-4, provou que a Hem se tornou uma área bem estabelecida de pesquisa desde o ICME 10 em 2004, com o TSG 29. A perspectiva adotada pelos pesquisadores no campo da Hem deve ser uma perspectiva cultural múltipla que permita, segundo Arzarello (2017, p. 12-13):

- a existência de diferentes posições epistemológicas e culturais relativas à matemática e sua relevância na cultura;

- a possível distância cultural das reformas curriculares propostas em relação à cultura matemática dos diferentes países;

- as relações com a cultura e as contribuições pessoais trazidas pelos alunos em sala de aula, tão relevantes para evitar a alienação dos alunos de seu ambiente cultural e para permitir que os alunos se envolvam na aprendizagem de uma maneira produtiva;

- a importância de ancorar o desenvolvimento profissional nas atividades dos professores (tradução livre) ${ }^{6}$.

O ICHME-5 ocorreu de 19 a 22 de setembro de 2017, na Universidade de Utrecht, na Holanda. Neste evento contabilizaram-se 40 trabalhos (BJARNADÓT'TIR et al., 2019). A mais recente conferência, ICHME-6, ocorreu no ano de 2019, entre os dias 16 e 20 de setembro, no Centre International de Rencontres Mathématiques (CIRM), na cidade de Marselha, França. O comitê científico internacional foi composto por Evelyne Barbin (Université de Nantes), Kristín Bjarnadóttir (University of Iceland), Fulvia Furinghetti (University of Genoa, Italy), Alexander Karp (Columbia University), Johan Prytz (Uppsala University),

\footnotetext{
6 "the existence of different epistemological and cultural positions concerning mathematics and its relevance in the culture; the possible, cultural distance of proposed curricular reforms from the mathematical culture of the different countries; the relationships with the culture and the personal contributions brought by the students in the classroom, so relevant to avoid the students' alienation from their cultural environment and to allow students to engage in learning in a productive way; the importance of anchoring professional development in teachers' activities" (ARZARELLO, 2017, p. 12-13).
} 
Gert Schubring (Bielefeld University). Os organizadores locais foram Evelyne Barbin (Université de Nantes) e Guillaume Moussard Barbin (Université de Nantes).

A partir da apresentação dos eventos científicos, e relacionando com a dinâmica do campo científico, Bourdieu (1976) menciona que a estrutura oculta do campo de produção, em particular os espaços de circulação, são capazes de produzir um efeito de autoconfirmação e auto reforço. As ressonâncias desses eventos científicos se aproximam de outras comunidades de pesquisadores interessados em pesquisar no campo da Hem.

González (2018) ao falar do aumento da comunidade de pesquisadores interessados em estudar a Hem, elenca mais quatro aspectos: a publicação do Handbook on the History of Mathematics Education (KARP; SCHUBRING, 2014 apud GONZÁLEZ, 2018), em que a Hem é examinada considerando várias épocas, civilizações, países e culturas. Outro aspecto são os dossiês temáticos em Hem, por exemplo, na Revista UNIÓN intitulada Historia Social de la Educación Matemática en Iberoamérica. A criação da Revista de História da Educação Matemática (HISTEMAT), foi outro aspecto, a divulgação de pesquisas entre pesquisadores, professores e outros interessados na “dimensão histórica, tanto da matemática como do seu ensino, dos resultados de investigações relativas à história da educação matemática e outras questões conexas" (p. 281, tradução livre)7 ${ }^{7}$ O autor também elenca o Congresso Ibero-americano de História da Educação Matemática (CIHEM), que "propicia o intercâmbio acadêmico, profissional e conceitual dos educadores matemáticos da América Latina, Espanha e Portugal que têm como assunto de interesse investigativo à história da educação matemática nestes espaços socio-geográficos" (p. 280, tradução livre) $)^{8}$.

A primeira edição do CIHEM ocorreu no ano de 2011 em Portugal, na Universidade da Beira Interior. Participaram 79 investigadores de diversos países

\footnotetext{
7 “dimensión histórica tanto de la matemática como de su enseñanza, de resultados de investigaciones relativas a la historia de la educación matemática y otros asuntos conexos" (GONZÁLEZ, 2018, p. 281).

8 "propicia el intercambio académico, profesional y conceptual de los educadores matemáticos de América Latina, España y Portugal que tienen como asunto de interés investigativo a la historia de la educación matemática en estos espacios sociogeográficos" (GONZÁLEZ, 2018, 208).
} 
como Brasil, Costa Rica, Espanha, México e Portugal, em que foram aceitos 53 comunicações e oito trabalhos em forma de pôster.

A realização do I Congresso Ibero-americano de História da Educação Matemática atendeu à necessidade de aprofundar o intercâmbio entre pesquisadores e a produção de conhecimento ligada à história da educação matemática na América Latina, na Espanha e em Portugal. O interesse pela temática tem crescido enormemente no âmbito da Educação Matemática nesses diversos países. Comissões internacionais, revistas com números especiais sobre o assunto, grupos de trabalho, de pesquisa e tantos outros indicadores mostram o quanto se justifica um evento desta natureza (MATOS; SARAIVA, 2011, p. 07).

O II CIHEM foi organizado pelo Departamento de Matemática Centro de Investigação em Educação e Estudos Avançados da IPN (Cinvestav-IPN), realizado em Cancun, no México, entre os dias 04 e 07 de novembro de 2013. Foram realizadas quatro palestras, quatro mesas e 58 comunicações (CANTORAL; VALENTE, 2013).

Em 2015 ocorreu o III CIHEM, entre os dias 04 e 07 de novembro, sendo realizado em Belém, estado do Pará, Brasil. Nesse evento ocorreram 13 conferências, uma mesa plenária com participação de oito conferencistas estrangeiros e seis brasileiros, e aprovados 128 trabalhos para serem apresentados (CHAQUIAM; MENDES; VALENTE, 2016). Segundo os organizadores do evento, as edições temáticas sobre a Hem "têm sido produzidas por conceituados periódicos nacionais e internacionais e, além dos inúmeros grupos de pesquisa que, embora criados mais recentemente, apresentam produção consistente" (CHAQUIAM; MENDES; VALENTE, 2016, p. 9).

Durante o evento, houve o lançamento da Revista HISTEMAT, vinculada à Sociedade Brasileira de História da Matemática (SBHMat). Esta revista aceita textos inéditos, resultantes de pesquisa sobre história da educação matemática, história e didática da matemática, história da matemática no ensino, e quaisquer produções que promovam o diálogo entre história, educação e matemática.

Em 2017, organizado pelo Centro de Estudios sobre la Memoria Educativa (CEME), da Faculdade de Educação da Universidade de Murcia, na Espanha, entre os dias 14 e 17 de novembro de 2017, ocorreu o IV CIHEM. E no 
ano de 2019, foi realizada a quinta edição na cidade de Bogotá na Colômbia, entre os dias 12 e 15 de novembro.

O IV CIHEM teve cinco objetivos: 1. Aprofundar o intercâmbio entre os investigadores da Hem na América Latina, Portugal e Espanha; 2. Difundir as diferentes perspectivas, temas e metodologias seguidas até agora; 3. Proporcionar a produção e divulgação de conhecimentos nesta área; 4. Promover a formação de grupos de trabalho e da colaboração entre os já existentes; e 5. Analisar o estado atual deste campo de investigação em apogeu e o seu futuro (GALLEGO et al., 2018, p. 10).

As conferências tiveram seis possibilidades de tópicos de estudos: T1. Avanços na investigação em Hem; T2. Metodologias de investigação em Hem; T3. Centros de estudo e documentação: experiências e organização; T4. Fontes para o estudo da Hem: manuais e livros para professores e estudantes, notebooks, os trabalhos dos alunos, exames, material didático, ilustrações; T5. A profissionalização dos educadores matemáticos: formação, seleção e carreira docente; e T6. Ciência e docência do conhecimento científico ao conhecimento pedagógico: gênese da Hem como disciplina e campo de investigação (GALLEGO et al., 2018, p. 10).

Segundo Chartier (2002), a produção, circulação e a apropriação de representações em formas de textos são examinadas a partir da relação entre as formas materiais e as práticas culturais. Assim, destacamos o seguinte excerto:

As ideias, apreendidas por meio da circulação das palavras que as designam, situadas nos seus enraizamentos sociais, pensadas na sua carga afectiva e emocional, tanto quanto no seu conteúdo intelectual, tornam-se assim, tal como os mitos ou os complexos de valores, uma dessas «forças colectivas pelas quais os homens vivem o seu tempo» e, portanto, uma das componentes da «psique colectiva» de uma civilização. Há aí como que um culminar da tradição dos Annales, na caracterização fundamentalmente psicológica da mentalidade colectiva e, simultaneamente, na redefinição do que deve ser a história das ideias reposta numa exploração de conjunto do mental colectivo (CHARTIER, 2002, p. 43, grifo do autor).

Entre as circulações de ideias e práticas por meio dos eventos e revistas científicas, destacamos o Encontro Nacional de Pesquisa em História da Educação Matemática (ENAPHEM), no Brasil, cuja primeira edição foi idealizada logo após o I CIHEM, realizado no ano de 2012 na Universidade Estadual do Sudoeste da Bahia 
(UESB), no município de Vitória da Conquista, Bahia, que contou com 19 palestras, 33 comunicações orais e 47 pôsteres. Vários pesquisadores brasileiros analisaram a pertinência de promover um evento nacional a fim de reunir toda comunidade de pesquisadores na área (SANTANA; VALENTE, 2012).

Segundo Pinto (2014), o I ENAPHEM “caracteriza-se como evento fundador que pela primeira vez, reúne a comunidade de pesquisadores de diferentes regiões do país para debater e trocar experiências investigativas de uma temática exclusiva, a história da educação matemática” (PINTO, 2014, p. 15).

ao buscarmos compreender a importância da participação dos inúmeros grupos de pesquisa que no Brasil têm contribuído para a escrita da história da educação matemática e que a partir de diferentes afiliações teóricas e para além da disputa de status acadêmico, deram visibilidade à pluralidade historiográfica do evento, a uma produção de histórias singulares (PINTO, 2014, p. 16).

Em 2014 ocorreu o II ENAPHEM na Universidade Estadual Paulista Júlio de Mesquita Filho (UNESP), campus Bauru em São Paulo, entre os dias 31 de outubro e 02 de novembro. Ao todo, foram realizadas duas mesas de abertura, duas sessões de memória, sete mesas redondas e 74 trabalhos apresentados em sessões coordenadas. Os eixos temáticos foram: 1. Histórias de formação de professores de matemática; 2. Histórias do ensino de matemática, em qualquer nível; 3. Histórias de artefatos didáticos relacionados e/ou voltados à educação matemática; 4. Histórias de grupos culturais ou comunidades de prática envolvidos com educação matemática; e 5. Histórias da produção científico-acadêmica em educação matemática (GARNICA; VALENTE, 2014).

O III ENAPHEM foi realizado na Universidade Federal do Espírito Santo (UFES), Campus São Mateus, entre os dias 31 de outubro a 02 de novembro de 2016. O evento contou com 179 membros entre pesquisadores e estudantes de pósgraduação. Compareceram ao evento 126 participantes de diversos estados brasileiros. Foram submetidos 93 trabalhos, sendo aprovados 74 comunicações orais e três mesas temáticas. A temática do evento foi "História da Educação Matemática e formação de professores" (DASSIE; COSTA, 2016).

No ano de 2018, entre os dias 14 e 17 de novembro, na Universidade Federal do Mato Grosso do Sul (UFMS), na cidade de Campo Grande, no estado do Mato 
Grosso do Sul, ocorreu o IV ENAPHEM. Foram apresentadas 78 comunicações científicas e 9 mesas redondas. O tema foi "Formação de Professores: história, cultura e política", segundo os organizadores, buscou-se ampliar e retomar as discussões em relação ao tema "formação de professores", e os temas relacionados a "cultura e política" são reflexões atuais sobre a formação de professores e a educação de forma geral, devem ser analisadas de forma conjunta (PINTO; DASSIE, 2018).

O V ENAPHEM, diferente das últimas edições, será virtual, diante da Pandemia COVID-19, e tem previsão para quatro encontros aos sábados, nos dias $07,14,21$ e 28 de novembro de 2020. A realização do evento conta com o apoio da UFRN.

A partir da realização das edições do ENAPHEM, são organizados livros a fim de reunir os resultados das reflexões presentes nos eventos, como uma contribuição para as pesquisas que investigam a Hem. Até o momento têm-se: História da Educação Matemática no Brasil (VALENTE, 2014), referente ao I ENAPHEM, realizado no ano de 2012; Pesquisa em História da Educação Matemática no Brasil: sob o signo da pluralidade (GARNICA, 2016), referente ao II ENAPHEM, realizado no ano de 2014; e História da Educação Matemática e Formação de Professores (DASSIE; COSTA, 2018), referente ao III ENAPHEM, realizado no ano de 2016. É importante destacar, que todos os livros possuem o apoio da SBHMat.

Outro espaço para a socialização das pesquisas que investigam a Hem, é o Seminário Internacional de Pesquisa em Educação Matemática (SIPEM). Segundo Coppe et al. (2018), os primeiros trabalhos apresentados que investigam a Hem no SIPEM, datam do II SIPEM, realizado no ano de 2003, com quatro pesquisas aprovadas, alocadas no GT 5 - História da Matemática e Cultura. Assim como, no IV SIPEM, vários pesquisadores da área da educação matemática começaram a pesquisar no campo da Hem, tendo em vista que seis dos 22 estudos apresentados pertenciam ao eixo da Hem. Os trabalhos no IV SIPEM utilizaram fontes documentais e orais e, em grande parte, explicitamente, contribuições da história cultural como perspectiva teórico-metodológica (COPPE et al., 2018).

Desta forma, no ano de 2018 ocorreu o VII SIPEM, inaugurando o GT15, intitulado "História da educação matemática". Segundo os coordenadores do GT15, 
Maria Cristina Araújo de Oliveira e Antonio Vicente Marafioti Garnica, a iniciativa para a criação do mesmo ocorreu no ano de 2016 em reunião na Sociedade Brasileira de Educação Matemática (SBEM), pela proposição de pesquisadores que atuam na Hem reunidos em diversos GPs (OLIVEIRA; GARNICA, 2018).

Além do que foi apresentado até o momento, cabe destacar os Dossiês Temáticos que contribuem para a circulação de ideias no campo da Hem no Brasil. O primeiro deles, foi publicado na revista Boletim de Educação Matemática (BOLEMA), no ano de 2010, em dois fascículos ${ }^{9}$. Os artigos relacionados ao primeiro volume são contribuições teórico-metodológica para as pesquisas na Hem, e buscam superar a dimensão teórica meramente, exemplificando com uma discussão de casos particulares para corroborar a argumentação desenvolvida (GOMES, 2010a). No segundo volume correspondente ao Dossiê, Gomes (2010b), menciona que os artigos foram divididos em três temáticas: o movimento da matemática moderna, os livros didáticos e as relações entre história da matemática e educação matemática.

No ano de 2016, a revista Diálogo Educacional, publicou o dossiê "História da Educação Matemática em Distintas Perspectivas"10. "Desse modo pretendemos de início, por meio desses estudos, ampliar o olhar do lugar da História da Educação Matemática" (PINTO; CORRÊEA, 2016). O Dossiê, foi composto por resultados parciais de pesquisas, estudos de distintas trajetórias de investigação, apresentando trabalhos inéditos.

Outro dossiê que destacamos é o publicado na revista Cadernos de História da Educação, no ano de 2017, intitulado “História da Educação Matemática e Formação de Professores que Ensinam Matemática"11. Esse dossiê resultou do

\footnotetext{
${ }^{9}$ Para maiores informações, disponível em: http://www.periodicos.rc.biblioteca.unesp.br/index.php/bolema/issue/view/1011, referente ao v. 25, n. $35 \mathrm{~A} \mathrm{e}$

http://www.periodicos.rc.biblioteca.unesp.br/index.php/bolema/issue/view/820, referente ao v. 25, n. 35B. Acesso em 20 out. 2019.

${ }^{10}$ Para maiores informações, disponível em: https://periodicos.pucpr.br/index.php/dialogoeducacional/issue/view/208, referente ao v. 16, n. 49. Acesso em 20 out. 2019.

${ }^{11}$ Para maiores informações, Cf. http://www.seer.ufu.br/index.php/che/issue/view/1529, referente ao v. 16, n. 3. Acesso em: 20 out. 2019.
} 
debate realizado no III ENAPHEM, a respeito do papel da história da educação matemática na formação de professores. "Este Dossiê, leva em consideração textos que sistematizam essas experiências em diferentes instituições. [...] em termos de análise da trajetória profissional dos professores que ensinam matemática, numa abordagem a ser tratada pela história da educação matemática" (VALENTE, 2017, p. 2).

Desta forma, há outros dossiês que contemplam as temáticas de investigação da Hem, como, por exemplo, o dossiê "Saberes Matemáticos no curso primário brasileiro: 1890-1970"12, organizado pelos professores David Antonio da Costa e Wagner Rodrigues Valente, publicado pela Revista Perspectiva em 2016. No ano de 2019, temos também dois dossiês voltados à Hem: um publicado na Revista Zetetiké, intitulado "Impressos para o ensino ou textos de referência e História da Educação Matemática: leituras e interpretações"13, e o dossiê "Saberes Matemáticos nas Escolas Normais: Brasil, França e Portugal (séculos XIX e XX)"14 na Revista Educação, organizado pelas professoras Andréia Dalcin e Elisabete Zardo Búrigo. Ou seja, há diversos dossiês que são publicados constantemente e apresentam diferentes perspectivas de investigação no campo da Hem.

\section{Algumas considerações}

Como vimos, um aspecto particular do campo científico é a intensa competição entre os pares na disputa da autoridade científica, constituindo-se como um espaço de lutas simbólicas entre dominantes e dominados. "É um campo de forças dotado de uma estrutura e também um espaço de conflitos pela manutenção ou transformação desse campo de forças” (BOURDIEU, 2001b, p. 52).

Para Bourdieu (2001b), "falar de campo significa romper com a ideia de que os cientistas formam um grupo unificado ou até homogêneo" (p. 67). Isto é, não

\footnotetext{
${ }^{12}$ Para maiores informações, Cf. https://periodicos.ufsc.br/index.php/perspectiva/issue/view/2328, referente ao v. 34, n. 1. Acesso em 08 nov. 2019.

${ }^{13} \mathrm{Cf}$. https://periodicos.sbu.unicamp.br/ojs/index.php/zetetike/issue/view/1589>, referente ao v. 27. Acesso em: 08 nov. 2019.

${ }^{14} \mathrm{Cf}$. http://revistaseletronicas.pucrs.br/ojs/index.php/faced/issue/view/1333, referente ao v. 42, n. 2. Acesso em: 08 nov. 2019.
} 
exclui os agentes possuírem características que os unem, separam, dividem e/ou os opõem. Assim, segundo Bourdieu (2001b), o campo científico busca uma autoridade, um reconhecimento e uma autonomia científica.

Ou seja, o campo é formado por tensões, busca um reconhecimento e legitimidade, ou melhor, um auto reforço e uma autoconfirmação, por meio da circulação de ideias, neste caso, em eventos e revistas científicas.

Finalizando, Bourdieu (2002) a respeito da circulação internacional das ideias, menciona que o sentido e a função de uma obra estrangeira são determinados mais pelo campo de chegada do que de origem. A transferência de um campo nacional para outro se faz por meio de operações sociais e de seleção, em particular, refletir para o que se traduz? O que se publica? Quem traduz? Quem publica?

No campo da Hem, pudemos observar o movimento de circulação internacional de ideias, iniciando com um pequeno grupo de pesquisadores interessados em investigar a Hem e aos poucos ocupando e criando espaços. Dessa forma, podemos identificar eventos e revistas científicas que contribuem para o reconhecimento e socialização do campo por meio das comunicações científicas, retomando Burke (2016), o meio mais eficaz para disseminação do conhecimento continua sendo o encontro com as pessoas.

\section{Referências}

ARZARELlO, F. Preface. In: BJARNADÓTTIR, K. et al. "Dig where you stand" 4. Proceedings of the fourth International Conference on the History of Mathematics Education. Rome: Edizioni Nuova Cultura, 2017.

BJARNADÓTTIR, K.; et al. "Dig where you stand" 5. Proceedings of the fifth International Conference on the History of Mathematics Education. Drukkerij Baas: Nieuwerkerk aan den Ijssel, 2019.

BJARNADÓTTIR, K. et al. "Dig where you stand" 2. Proceedings of the second international conference on the history of mathematics education. Lisbon, Caparica: UIED, 2012.

BJARNADÓTTIR, K. et al. "Dig where you stand" 4. Proceedings of the fourth International Conference on the History of Mathematics Education. Rome: Edizioni Nuova Cultura, 2017. 
BJARNADÓT'TIR, K. et al. "Dig where you stand" 3. Proceedings of the third international conference on the history of mathematics education, Uppsala: Department of Education, Uppsala University, 2015.

BJARNADÓTTIR, K.; FURINGHETTI, F.; SCHUBRING, G. "Dig where you stand". Proceedings of the conference "On-going research in the history of mathematics education". Reykjavik: University of Iceland, School of Education, 2009.

BOURDIEU, P. Os usos sociais da ciência: por uma sociologia clínica do campo científico. São Paulo: Unesp, 2004.

BOURDIEU, P. Les conditions sociales de la circulation internationale des idées. In: Actes de la recherche en sciences sociales. v. 145, décembre 2002. p. 3-8. (La circulation internationale des idées).

BOURDIEU, P. Meditações pascalianas. Rio de Janeiro: Bertrand Brasil, 2001a.

BOURDIEU, P. Para uma sociologia da ciência. Lisboa: Ed. 70, 2001 b.

BOURDIEU, P. Le champ scientifique. In: Actes de la recherche en sciences sociales, v. 2, n. 2-3, p. 88-104, juin 1976. (La production de l’idéologie dominante).

BRITO, A. J.; MIORIM, M. A. A institucionalização da História da Educação Matemática. In: ANTONIO, V.; GARNICA, M. (Org.). Pesquisa em História da Educação Matemática no Brasil. 1ed. São Paulo: Livraria da Física Editora, 2016. p. 67-92.

BUENO, W. C. Comunicação científica e divulgação científica: aproximações e rupturas conceituais. Informação \& Informação, [S.1.], v. 15, n. 1esp, p. 1-12, dez. 2010.

BURKE, P. O que é história do conhecimento? São Paulo: Ed. Unesp, 2016.

CANTORAL, R.; VALENTE, W. R. Congresso Ibero-americano de História de Educação Matemática, 2013, Cancun. Anais... Cacun, 2013.

CHAQUIAM, M.; MENDES, I. A.; VALENTE, W. R. Congresso Ibero-americano de História de Educação Matemática, 2016, Belém. Anais... Belém, 2016.

CHARTIER, R. Capítulo 1. História intelectual e história das mentalidades. In: CHARTIER, R. A História Cultural entre práticas e representacõoes. Col. Memória e sociedade. Trad. Maria Manuela Galhardo. Rio de Janeiro: Bertrand Brasil, 2002. p. 29-67.

COPPE, C. et al. History of Mathematics and Culture: Moments and Movements in Brazilian Mathematics Education. In: RIBEIRO, A. J. et al. (Org.). Mathematics Education in Brazil Panorama of Current Research. 1ed. Switzerland: Springer, 2018. p. 1-278.

COSTA, D. A.; VALENTE, W. Saberes Matemáticos no curso primário brasileiro 1890 1970. Perspectiva, Florianópolis, v. 34, n.1, 2016.

DALCIN, A.; BÚRIGO, E. Z. Saberes matemáticos nas escolas normais: Brasil, França e Portugal (séculos XIX e XX). Revista Educação, Porto Alegre, v. 42, n.2, 2019. 
DASSIE, B. A.; COSTA, D. A. História da Educação Matemática e Formação de Professores. 1. ed. São Paulo: Editora Livraria da Física, v. 1. 241p. 2018.

DASSIE, B. A.; COSTA, D. A. Encontro Nacional de Pesquisa em História da Educação Matemática (3.: 2016: São Mateus, ES). Anais... 2016

FURINGHET'TI, F.; KARP, A. (Org.). Researching the History of Mathematics Education: ICME-13 Monographs. 1. ed. Cham: Springer International Publishing, 2018.

GALLEGO, D. C. et al. Congresso Ibero-americano de História de Educação Matemática, 2018, Murcia. Anais do IV... Murcia, 2018.

GARNICA, A. V. M. Pesquisa em História da Educação Matemática no Brasil: sob o signo da pluralidade. 01. ed. São Paulo: Editora da Física, v. 1500. 213p. 2016.

GARNICA, A. V. M.; VALENTE, W. R. Encontro Nacional de Pesquisa em História da Educação Matemática, 2014, Bauru. Anais... 2014.

GOMES, M. L. M. História da Educação Matemática: a propósito da edição temática do BOLEMA. Bolema, Boletim de Educação Matemática, Rio Claro, Impresso, v. 23, n. 35A, p. 1-21, 2010a.

GOMES, M. L. M. O segundo fascículo da edição temática do BOLEMA sobre História da Educação Matemática. Bolema, Boletim de Educação Matemática, Rio Claro, Impresso, v. 23, n. 35B, p. v-x, 2010b.

GONZÁLEZ, F. E. Historia de la Educación Matemática en Latinoamérica: 10 claves para su comprensión. Unión: revista iberoamericana de educación matemática, n. 58, p. 279-305, abr. 2018.

HOFFMANN, Y. T.; COSTA, D. A. História da educação matemática: um campo de lutas. Campo Grande, 2019. In: Anais... 2019. p. 1-13.

MATOS, J. M.; SARAIVA, M.. Congresso Ibero-americano de História de Educação Matemática (1.: 2011: Covilhã: Portugal). In: Anais do I Congresso Ibero-americano de História da Educação Matemática, 2011.

OLIVEIRA, M. C. A; GARNICA, A. V. M. Relatório do GT 15 - História da educação matemática. In: Relatório VII SIPEM. Seminário Internacional de Pesquisa em Educação Matemática, Foz do Iguaçu, Paraná, 4 a 8 de novembro de 2018. p. 155-164.

PINTO, N. B. A Educação Matemática Brasileira e a Realização do Primeiro Encontro Nacional de Pesquisas em História da Educação Matemática. Da importância do I ENAPHEM. In: VALENTE, W. R. (Org.). História da Educação Matemática no Brasil. Problemáticas de pesquisa, fontes. referências teórico-metodológicas e histórias elaboradas. 1ed.São Paulo: Editora Livraria da Física, 2014. p. 13-29.

PINTO, N. B.; CORREA, R. L. T. História da educação matemática em distintas perspectivas. Editorial. Revista Diálogo Educacional, Curitiba, v. 16, n. 49, 2016. 
PINTO, T. P.; DASSIE, B. A. Encontro Nacional de Pesquisa em História da Educação Matemática, 2018, Campo Grande. Anais... Campo Grande, 2018.

SANTANA, C. C.; VALENTE, W. R. Encontro Nacional de Pesquisa em História da Educação Matemática, 2012, Vitória da Conquista. Anais... Vitória da Conquista, 2012.

SCHUBRING, G. Editorial. In: The International Journal for the History of Mathematics Education, v. 1, n. 1, 2006.

TARGINO, M. DAS G. COMUNICAÇÃO CIENTÍFICA: uma revisão de seus elementos básicos. Informação \& Sociedade: Estudos, v. 10, n. 2, jan. 2000.

VALENTE, W. R. História da Educação Matemática e Formação de Professores que Ensinam Matemática. Cadernos De História Da Educação (Online), v. 16, n. 3, p. 608-609, 2017.

VALENTE, W. R. História da Educação Matemática no Brasil. 1. ed. São Paulo: Editora Livraria da Física, v. 1, 2014. 331p.

RECEBIDO: $21 / 03 / 2020$

APROVADO: $24 / 07 / 2020$

RECEIVED: 03/21/2020

APPROVED: 07/24/2020

RECIBIDO: $21 / 03 / 2020$

APROBADO: $24 / 07 / 2020$ 


\section{Anexo}

Quadro: Grupos de Pesquisas que investigam a Hem no Brasil

\begin{tabular}{|c|c|c|c|}
\hline Nome do GP & Instituição & $\begin{array}{l}\text { Ano de } \\
\text { Criação }\end{array}$ & Líder \\
\hline $\begin{array}{c}\text { História, Filosofia e Educação Matemática } \\
\text { (HIFEM) }\end{array}$ & UNESP & 1996 & $\begin{array}{l}\text { Arlete de Jesus Brito e } \\
\text { Andreia Dalcin }\end{array}$ \\
\hline $\begin{array}{c}\text { Grupo de Pesquisa de História da Educação } \\
\text { Matemática (GHEMAT) }\end{array}$ & UNIFESP & 2000 & $\begin{array}{l}\text { Wagner Rodrigues Valente e } \\
\text { Neuza Bertoni Pinto }\end{array}$ \\
\hline $\begin{array}{l}\text { História Oral e Educação Matemática } \\
\text { (GHOEM) }\end{array}$ & UNESP & 2002 & $\begin{array}{l}\text { Antonio Vicente Marafioti } \\
\text { Garnica e Heloisa da Silva }\end{array}$ \\
\hline $\begin{array}{c}\text { História da Educação Matemática: aspectos } \\
\text { históricos, curriculares e culturais } \\
\text { (GHisemahcc) }\end{array}$ & UFOP & 2008 & $\begin{array}{l}\text { Marger da Conceição Ventura } \\
\text { Viana e Milton Rosa }\end{array}$ \\
\hline $\begin{array}{l}\text { Núcleo de Pesquisas e Estudos em Educação } \\
\text { Matemática (NUPEm) }\end{array}$ & UFU & 2009 & $\begin{array}{l}\text { Cristiane Coppe de Oliveira e } \\
\text { Vlademir Marim }\end{array}$ \\
\hline $\begin{array}{l}\text { Grupo de Estudos e Pesquisa em Educação } \\
\text { Matemática (GEPEM IFSC) }\end{array}$ & IFSC & 2011 & $\begin{array}{l}\text { Elenita Eliete de Lima Ramos e } \\
\text { Jeremias Stein Rodriguês }\end{array}$ \\
\hline $\begin{array}{c}\text { História da Educação Matemática em } \\
\text { Pesquisa (HEMEP) }\end{array}$ & UFMS & 2011 & $\begin{array}{l}\text { Luzia Aparecida de Souza e } \\
\text { Thiago Pedro Pinto }\end{array}$ \\
\hline Educação e História da Matemática & UECE & 2013 & $\begin{array}{l}\text { Ana Carolina Costa Pereira e } \\
\text { Isabelle Coelho da Silva }\end{array}$ \\
\hline $\begin{array}{l}\text { Grupo Potiguar de Estudos e Pesquisas em } \\
\text { História da Educação Matemática (GPEP) }\end{array}$ & UFRN & 2013 & $\begin{array}{l}\text { Liliane dos Santos Gutierre e } \\
\text { Fernando Guedes Cury }\end{array}$ \\
\hline $\begin{array}{l}\text { Grupo Rondoniense de Estudos e Pesquisas } \\
\text { em Educação Matemática (GROEPEM) }\end{array}$ & UNIR & 2015 & $\begin{array}{l}\text { Emerson da Silva Ribeiro e } \\
\text { Kecio Gonçalves Leite }\end{array}$ \\
\hline $\begin{array}{c}\text { Estratégias de Ensino para Educação Básica } \\
\text { e Profissional }\end{array}$ & IFSul & 2017 & Malcus Cassiano Kuhn \\
\hline $\begin{array}{l}\text { Grupo de Pesquisa de História da Educação } \\
\text { Matemática (GHEMAT / PR) }\end{array}$ & UTFPR & 2017 & $\begin{array}{c}\text { Barbara Winiarski Diesel } \\
\text { Novaes e Mariliza Simonete } \\
\text { Portela }\end{array}$ \\
\hline $\begin{array}{c}\text { Grupo de Pesquisa de História da Educação } \\
\text { Matemática (GHEMAT / SC) }\end{array}$ & UFSC & 2017 & $\begin{array}{l}\text { David Antonio da Costa e } \\
\text { Iara Zimmer }\end{array}$ \\
\hline $\begin{array}{c}\text { História da Matemática e Educação } \\
\text { Matemática }\end{array}$ & UFAL & 2017 & Viviane de Oliveira Santos \\
\hline $\begin{array}{l}\text { Grupo de Estudo e Pesquisa em História da } \\
\text { Educação Matemática (GEPHEME-RO) }\end{array}$ & UNIR & 2017 & $\begin{array}{l}\text { Enoque da Silva Reis e Luiz } \\
\text { Carlos Pais }\end{array}$ \\
\hline $\begin{array}{l}\text { Grupo de Pesquisa Educação Matemática e } \\
\text { Diversidade Cultural (GPEMDiC) }\end{array}$ & UESC & 2018 & $\begin{array}{l}\text { Jurema Lindote Botelho } \\
\text { Peixoto e Zulma Elizabete de } \\
\text { Freitas Madruga }\end{array}$ \\
\hline $\begin{array}{c}\text { Grupo de Pesquisa em História da Educação } \\
\text { Matemática }\end{array}$ & IFMG & 2018 & Thiago Neves Mendonça \\
\hline $\begin{array}{c}\text { Grupo de Pesquisas e Estudos em Educação } \\
\text { Matemática (GPEEM) }\end{array}$ & IFESP & 2018 & José Paulino Filho \\
\hline
\end{tabular}

